\title{
TERMOGRAFIA DIGITAL POR INFRAVERMELHO DO ESCROTO E QUALIDADE DO SÊMEN EM TOUROS NELORE (BOS TAURUS INDICUS)
}

Felipe Rydygier de Ruediger ${ }^{1}$, Marcelo George Mungai Chacur ${ }^{2}$, Eunice Oba ${ }^{3}$, Alcides Ramos Amorim $^{3}$, Camila Dutra de Souza ${ }^{1}$

${ }^{1,2}$ Universidade do Oeste Paulista - UNOESTE. Mestrado em Ciência Animal, Presidente Prudente - SP. ${ }^{3}$ Universidade Estadual Paulista - FMVZ/UNESP. Docente, Botucatu -SP. E-mail: chacur@unoeste.br

\section{RESUMO}

Objetivou-se estudar a relação da temperatura do escroto sobre a qualidade seminal pelo uso da termografia por infravermelho em touros Nelore criados extensivamente. Realizaram-se termografia escrotal e colheita de sêmen. Os dados climáticos foram coletados por meio de globo termômetro. Os termogramas do escroto foram obtidos por meio de termografia digital de infravermelho. Efetuaram-se colheitas de sêmen, por meio de eletroejaculação para analise das características quantitativas e qualitativas. Para os dados, utilizou-se análise de variância e posteriormente aplicou-se o teste de Tukey a $5 \% .(p<0,05)$ entre animais para vigor espermático, motilidade espermática entre animais e entre colheitas, concentração espermática, espermatozoides totais e espermatozoides viáveis totais entre grupos, entre colheitas para as temperaturas do escroto e para os dados climáticos. Concluiu-se que as temperaturas da superfície do escroto e os fatores climáticos influenciaram na qualidade do sêmen. A termografia é recomendada como exame complementar na avaliação reprodutiva de touros.

Palavras-chave: fatores climáticos; termograma escrotal; touro zebu; sêmen.

\section{SCROTUM DIGITAL INFRARED THERMOGRAPHY AND SEMEN QUALITY IN NELORE BULLS (BOS TAURUS INDICUS)}

\begin{abstract}
Aimed to study the relationship between scrotal temperature on semen quality by use of infrared thermography in Nelore bulls bred extensively. There were scrotal thermography and semen collection. Climatic data were collected by means of globe thermometer. The thermograms of the scrotum were obtained by digital infrared thermography. We carried out ejaculates through electroejaculation for analysis of quantitative and qualitative characteristics. For the data, we used analysis of variance and subsequently applied the Tukey test at $5 \% .(p<0.05)$ between animals for sperm vigor, sperm motility between animals and between crops, sperm concentration, total sperm and total viable sperm between groups, between harvests for the scrotum temperatures and climate data. It was concluded that the surface temperatures of the scrotum and climatic factors influence the quality of semen. Thermography is recommended as a complementary test in the evaluation of reproductive bulls.
\end{abstract}

Keywords: climatic factors; scrotal thermogram; semen; zebu bull. 


\section{INTRODUÇÃO}

Para otimizar a função reprodutiva para monta natural ou inseminação artificial, os touros devem produzir um grande número de espermatozoides morfologicamente normais e o conhecimento sobre os testículos dos bovinos e como eles são avaliados é de grande importância (KASTELIC, 2014).

Diversos fatores afetam a reprodução animal, entre eles a temperatura ambiente e umidade relativa do ar (HORN et al., 1997). De acordo com Kastelic (2001), uma moderada elevação da temperatura testicular em touros submetidos à insulação escrotal, reduz de forma drástica a produção espermática, a motilidade progressiva e a quantidade de espermatozoides vivos por ejaculado, e aumenta a porcentagem de espermatozoides morfologicamente anormais.

Manter a temperatura dos testículos do touro entre $4-5^{\circ} \mathrm{C}$ abaixo da corporal é essencial para a produção eficaz de sêmen. Efeitos adversos da temperatura testicular elevada tem sidos relatados na produção de sêmen, na sua qualidade e na fertilidade de ruminantes (COULTER et al., 1988; BRITO et al., 2004). Mecanismos locais, como a troca de calor por contracorrente, regulação do fluxo sanguíneo, posição dos testículos e sudorese, desempenham um papel importante na manutenção da temperatura testicular (GABALDI; WOLF, 2002; BRITO et al., 2004).

A termografia por infravermelho é um exame não invasivo com elevada acurácia. A temperatura da superfície escrotal é correlacionada com a temperatura testicular, fornecendo informações detalhadas sobre a capacidade do touro em manter a temperatura testicular (COULTER et al., 1988).

Os zebuínos predominam a composição do rebanho brasileiro, sendo exigidos para a sua eficiência reprodutiva. $\mathrm{O}$ estudo do emprego da termografia digital por infravermelho em touros zebuínos tem sido pouco explorado como recurso diagnóstico devido ao pequeno número de artigos publicados. Objetivou-se estudar a relação da temperatura do escroto sobre a qualidade seminal pelo uso da termografia digital por infravermelho em touros Nelore criados extensivamente.

\section{METODOLOGIA}

O experimento foi aprovado pelo Comitê de Ética e Uso de Animais em Experimentação (CEUA) da Universidade do Oeste Paulista, Presidente Prudente-SP, sob o protocolo número 1700 .

Este foi realizado de outubro a dezembro de 2012 em uma propriedade rural pertencente ao município de Piquerobi-SP,

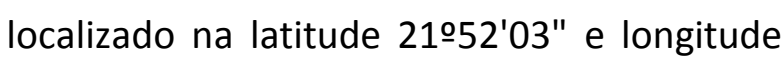
51ㄴㄱ'43" com precipitação média de 1.300 
$\mathrm{mm} / \mathrm{ano}$, temperatura média anual de $28^{\circ} \mathrm{C}$, clima quente com inverno seco. Foram utilizados 6 touros Nelore com idades entre 33 e 35 meses, criados extensivamente, em pasto de Brachiaria decumbens, com acesso ao sal mineral e água ad libitum.

Os dados climáticos no período do experimento foram coletados por meio de globo termômetro (InstruTemp ${ }^{\circledR}$, ITWTG2000) no período matutino, avaliando a temperatura global com termômetro úmido (WBGT), temperatura de globo preto (TG), temperatura do ar (TA) e umidade relativa do $\operatorname{ar}(\mathrm{UR})$.

Foram aferidas temperaturas da superfície do escroto por meio de termografia digital de infravermelho (Termovisor FLIR ${ }^{\circledR}$ E-40), sendo o foco emissor do aparelho direcionado na porção caudal do escroto de cada touro e orientado, perpendicularmente, a $1 \mathrm{~m}$ de distância. As imagens foram analisadas pelo software FLIR Tools $^{\circledR}$ versão 3.1.13080.1002 para se obter as temperaturas das regiões em 5 pontos da superfície escrotal, dos lados direito (D) e esquerdo (E): colo do escroto (TD1 e TE1), terços dorsal (TD2 e TE2), médio (TD3 e TE3), e ventral (TD4 e TE4) dos testículos e cauda do epidídimo (TD5 e TE5) (LUNSTRA; COULTER, 1997). Após foi aferida a temperatura retal (TR) com termômetro clínico digital.

Foram efetuadas colheitas de sêmen dos touros, por meio da eletroejaculação
(Autoejac $^{\circledR}$, Neovet, Brasil), com aparelho automático. As amostras de sêmen foram mantidas em banho-maria, entre 32 e $35^{\circ} \mathrm{C}$, para as análises imediatas da motilidade espermática progressiva, vigor espermático e turbilhão. Com posterior diluição do sêmen em formol salino tamponado (1:100), para obtenção da concentração espermática em câmara de Neubauer e da morfologia espermática frente à avaliação de 200 células com microscopia óptica de contraste de fase. Os touros foram classificados segundo as avaliações clínicas e espermáticas para efeito de seleção para monta natural, segundo as normas do Colégio Brasileiro de Reprodução Animal (CBRA, 2013).

Foram realizadas colheitas de sangue por venopunção jugular, junto a cada colheita de sêmen, o sangue foi centrifugado a $1500 \mathrm{~g} / 15$ minutos e o soro armazenado em criotubos a $-20^{\circ} \mathrm{C}$, para posterior dosagem de testosterona, pelo método de radioimunoensaio (RIA), utilizando-se kit comercial (DPC - Medlab ${ }^{\circledR}$ ).

O delineamento estatístico utilizado foi inteiramente ao acaso, os dados foram avaliados pela análise de variância e posteriormente aplicou-se o teste de Tukey a $5 \%$, com o "software" SAS (2005).

\section{RESULTADOS}

Os dados da morfometria testicular e epididimária e da circunferência escrotal 
observados estão dentro do padrão anatômico segundo CBRA (2013).

Para as características seminais houve diferença significativa $(P<0,05)$ entre animais para vigor espermático, sendo o animal 6 superior aos demais. Para motilidade espermática $(P<0,05)$ entre animais e entre colheitas, sendo os animais 5 e 6 , e a colheita 2 com melhores médias. Para o vigor espermático, houve diferença estatística $(P<0,05)$ entre animais, tendo o animal 6 melhor vigor (4). Para a concentração espermática, espermatozoides totais e espermatozoides viáveis totais $(P<0,05)$ entre grupos, com o grupo 2 tendo melhor média para estas variáveis. Nas variáveis defeitos maiores, defeitos menores e defeitos totais $(p<0,05)$ entre animais, tendo a menor porcentagem $\mathrm{o}$ animal $1 \mathrm{em}$ todas as características (TABELA 1).

Tabela 1. Valores médios, desvio padrão e análise de variância para as características qualitativas e quantitativas do sêmen dos touros Nelore.

\begin{tabular}{|c|c|c|c|c|c|c|c|c|c|c|c|}
\hline & $\mathrm{VOL}^{1}$ & $\mathrm{ASP}^{2}$ & TUR $^{3}$ & MOT $^{4}$ & $\mathrm{VIG}^{5}$ & $\operatorname{cONC}^{6}$ & ESPTO $^{7}$ & ESVIT $^{8}$ & $\mathrm{DMA}^{9}$ & $\mathrm{DME}^{10}$ & DTO $^{11}$ \\
\hline \multicolumn{12}{|l|}{$\begin{array}{l}\text { Média } \\
\text { colheitas }\end{array}$} \\
\hline \multirow{2}{*}{1} & 5.25 & 1.83 & 1.50 & 65.00 & 3.33 & 13.91 & 75018 & 48.30 & 7.38 & \multirow{2}{*}{$5.90 \mathrm{~A}$} & 13.28 \\
\hline & $A$ & $A$ & $A$ & $A B$ & $A$ & B & 15.04 B & B & $A$ & & $A$ \\
\hline \multirow{2}{*}{2} & 3.66 & 2.16 & 1.66 & 80.00 & 3.33 & 50.91 & 254.42 & 192.91 & 9.17 & \multirow{2}{*}{$6.35 \mathrm{~A}$} & 15.52 \\
\hline & A & A & $A$ & A & A & A & A & A & A & & $A$ \\
\hline \multirow{2}{*}{3} & 5.33 & 1.83 & 1.33 & 65.83 & 2.50 & 19.00 & $102 \wedge \mathrm{P}$ & 72.25 & 6.67 & \multirow{2}{*}{$5.96 \mathrm{~A}$} & 12.64 \\
\hline & $A$ & $A$ & $A$ & $A B$ & A & B & & $A B$ & $A$ & & $A$ \\
\hline \multirow{2}{*}{4} & 4.91 & 1.33 & 1.50 & 58.33 & 3.16 & 17.75 & 72.54 & 44.84 & 6.10 & \multirow{2}{*}{$6.59 \mathrm{~A}$} & 12.70 \\
\hline & $A$ & $\mathrm{~A}$ & $A$ & B & $A$ & B & $A B$ & B & $\mathrm{A}$ & & $A$ \\
\hline \multirow{2}{*}{5} & 6.16 & 1.83 & 1.50 & 73.33 & 3.33 & 24.16 & 154.38 & 115.20 & 6.66 & \multirow{2}{*}{$7.09 \mathrm{~A}$} & 13.75 \\
\hline & $A$ & $A$ & $A$ & $A B$ & $A$ & B & B & $A B$ & $A$ & & $A$ \\
\hline \multirow{2}{*}{6} & 5.08 & 1.83 & 1.50 & 73.33 & 3.50 & 16.00 & 83.21 & 60.22 & 8.32 & \multirow{2}{*}{$4.65 \mathrm{~A}$} & 12.97 \\
\hline & $A$ & $A$ & $A$ & $A B$ & $A$ & B & $A B$ & B & $A$ & & $A$ \\
\hline \multicolumn{12}{|l|}{$\begin{array}{c}\text { Média } \\
\text { animais }\end{array}$} \\
\hline \multirow{2}{*}{1} & 6.58 & 1.50 & 0.66 & 65.00 & 2.66 & 15.75 & 174.17 & 114.78 & 4.10 & \multirow{2}{*}{$2.73 \mathrm{C}$} & 6.84 \\
\hline & $A$ & $A$ & $B C$ & $A B$ & $B C$ & $A$ & $A$ & $A$ & B & & B \\
\hline \multirow{2}{*}{2} & 4.50 & 1.66 & 1.66 & 72.50 & 3.33 & 23.33 & $9525 A$ & 71.18 & 10.14 & \multirow{2}{*}{$3.82 \mathrm{C}$} & 13.97 \\
\hline & $A$ & $A$ & $A B C$ & $A B$ & $A B C$ & A & $95.25 \mathrm{~A}$ & $A$ & A & & $A$ \\
\hline & 4.75 & 1.33 & 0.00 & 56.66 & 2.50 & 21.58 & 102.75 & 65.23 & 10.39 & 5.12 & 15.51 \\
\hline & $A$ & $A$ & C & B & C & A & A & A & A & $B C$ & A \\
\hline & 5.58 & 1.83 & 0.83 & 60.00 & 2.83 & 27.66 & 153.04 & 103.93 & 5.99 & 9.64 & 15.63 \\
\hline & $A$ & $\mathrm{~A}$ & $\mathrm{BC}$ & B & $A B C$ & $A$ & A & A & $A B$ & $A B$ & $A$ \\
\hline & 4.33 & 2.16 & 2.50 & 80.00 & 3.83 & 29.08 & 105.63 & 84.94 & 6.48 & 10.21 & 16.69 \\
\hline & $A$ & $\mathrm{~A}$ & $A B$ & A & $A B$ & A & A & A & $A B$ & A & $A$ \\
\hline \multirow[b]{2}{*}{6} & 4.66 & 2.33 & 3.33 & 81.66 & 4.00 & 24.33 & 111.75 & 93.65 & 7.20 & 5.02 & 12.23 \\
\hline & A & $A$ & A & A & $A$ & A & A & A & $A B$ & $B C$ & $A B$ \\
\hline
\end{tabular}


Na Tabela $2(P<0,05)$ entre colheitas $E(P<0,05)$ entre animais para T2D e T3D. para T1E, T2E, T3E, T4E, T1D, T2D, T3D e TR.

Tabela 2. Valores médios, desvio padrão e análise de variância das temperaturas $\left({ }^{\circ} \mathrm{C}\right)$ do escroto aferidas por termografia de infravermelho e temperatura retal em touros criados extensivamente.

\begin{tabular}{|c|c|c|c|c|c|c|c|c|c|c|c|}
\hline & T1E & $\mathrm{T} 2 \mathrm{E}$ & T3E & T4E & T5E & T1D & $\mathrm{T} 2 \mathrm{D}$ & T3D & T4D & T5D & TR \\
\hline $\begin{array}{c}\text { Média } \\
\text { colheit } \\
\text { as }\end{array}$ & & & & & & & & & & & \\
\hline 1 & $\begin{array}{c}32.10 \\
B\end{array}$ & $\begin{array}{c}31.13 \\
B\end{array}$ & $\begin{array}{c}30.48 \\
\text { B }\end{array}$ & $\begin{array}{c}30.11 \\
\text { B }\end{array}$ & $\begin{array}{c}29.11 \\
\text { A }\end{array}$ & $\begin{array}{c}30.80 \\
\text { B }\end{array}$ & $\begin{array}{c}30.33 \\
\text { B }\end{array}$ & $\begin{array}{c}30.21 \\
\text { B }\end{array}$ & $\begin{array}{c}29.91 \\
\text { A }\end{array}$ & $\begin{array}{c}29.38 \\
A\end{array}$ & $\begin{array}{c}39.18 \\
B C\end{array}$ \\
\hline 2 & $\begin{array}{c}33.56 \\
\text { BA }\end{array}$ & $\begin{array}{c}33.23 \\
A\end{array}$ & $\begin{array}{c}32.40 \\
\text { A }\end{array}$ & $\begin{array}{c}31.81 \\
\mathrm{~A}\end{array}$ & $\begin{array}{c}30.95 \\
\text { A }\end{array}$ & $\begin{array}{c}33.28 \\
\text { A }\end{array}$ & $32.26 \mathrm{~A}$ & $\begin{array}{c}31.48 \\
\text { BA }\end{array}$ & $\begin{array}{c}31.01 \\
A\end{array}$ & $\begin{array}{c}30.30 \\
\mathrm{~A}\end{array}$ & $38.98 \mathrm{C}$ \\
\hline 3 & $\begin{array}{c}33.56 \\
\text { BA }\end{array}$ & $\begin{array}{c}33.03 \\
A\end{array}$ & $\begin{array}{c}32.21 \\
A\end{array}$ & $\begin{array}{c}31.61 \\
\text { BA }\end{array}$ & $\begin{array}{c}30.56 \\
\text { A }\end{array}$ & $\begin{array}{c}32.63 \\
\text { BA }\end{array}$ & $32.63 \mathrm{~A}$ & $\begin{array}{c}31.81 \\
\mathrm{~A}\end{array}$ & $\begin{array}{c}31.16 \\
A\end{array}$ & $\begin{array}{c}30.25 \\
A\end{array}$ & $\begin{array}{c}39.25 \\
\text { BAC }\end{array}$ \\
\hline 4 & $\begin{array}{c}32.95 \\
\text { BA }\end{array}$ & $\begin{array}{c}32.10 \\
\text { BA }\end{array}$ & $\begin{array}{c}31.28 \\
\text { BA }\end{array}$ & $\begin{array}{c}30.65 \\
\text { BA }\end{array}$ & $\begin{array}{c}29.53 \\
A\end{array}$ & $\begin{array}{c}32.48 \\
\text { BA }\end{array}$ & $\begin{array}{c}31.41 \\
\text { BA }\end{array}$ & $\begin{array}{c}30.75 \\
\text { BA }\end{array}$ & $\begin{array}{c}30.06 \\
A\end{array}$ & $\begin{array}{c}29.28 \\
A\end{array}$ & $\begin{array}{c}39.40 \\
\text { BA }\end{array}$ \\
\hline 5 & $\begin{array}{c}34.23 \\
A\end{array}$ & $\begin{array}{c}33.31 \\
A\end{array}$ & $\begin{array}{c}32.10 \\
A\end{array}$ & $\begin{array}{c}31.95 \\
A\end{array}$ & $\begin{array}{c}30.90 \\
\mathrm{~A}\end{array}$ & $\begin{array}{c}33.51 \\
\text { A }\end{array}$ & $32.33 \mathrm{~A}$ & $\begin{array}{c}31.70 \\
A\end{array}$ & $\begin{array}{c}31.08 \\
A\end{array}$ & $\begin{array}{c}30.58 \\
A\end{array}$ & $38.98 \mathrm{C}$ \\
\hline 6 & $\begin{array}{c}33.08 \\
\text { BA }\end{array}$ & $\begin{array}{c}33.23 \\
A\end{array}$ & $\begin{array}{c}31.48 \\
\text { BA }\end{array}$ & $\begin{array}{c}31.93 \\
A\end{array}$ & $\begin{array}{c}30.96 \\
A\end{array}$ & $\begin{array}{c}31.96 \\
\text { BA }\end{array}$ & $32.58 \mathrm{~A}$ & $\begin{array}{c}31.76 \\
A\end{array}$ & $\begin{array}{c}31.31 \\
A\end{array}$ & $\begin{array}{c}30.78 \\
A\end{array}$ & $39.61 \mathrm{~A}$ \\
\hline $\begin{array}{l}\text { Média } \\
\text { animai }\end{array}$ & & & & & & & & & & & \\
\hline 1 & $\begin{array}{c}32.65 \\
\text { A }\end{array}$ & $\begin{array}{c}32.08 \\
\text { A }\end{array}$ & $\begin{array}{c}31.15 \\
A\end{array}$ & $\begin{array}{c}30.56 \\
A\end{array}$ & $\begin{array}{c}29.76 \\
A\end{array}$ & $\begin{array}{c}31.85 \\
A\end{array}$ & $\begin{array}{c}31.58 \\
A B C\end{array}$ & $\begin{array}{c}30.73 \\
\text { B }\end{array}$ & $\begin{array}{c}30.30 \\
A\end{array}$ & $\begin{array}{c}29.91 \\
\text { A }\end{array}$ & $39.15 \mathrm{~A}$ \\
\hline 2 & $\begin{array}{c}33.90 \\
A\end{array}$ & $\begin{array}{c}32.81 \\
\text { A }\end{array}$ & $\begin{array}{c}31.73 \\
A\end{array}$ & $\begin{array}{c}31.38 \\
A\end{array}$ & $\begin{array}{c}30.51 \\
\text { A }\end{array}$ & $\begin{array}{c}32.95 \\
\text { A }\end{array}$ & $33.03 \mathrm{~A}$ & $\begin{array}{c}32.28 \\
A\end{array}$ & $\begin{array}{c}31.13 \\
\text { A }\end{array}$ & $\begin{array}{c}29.86 \\
\text { A }\end{array}$ & $\begin{array}{c}39.15 \\
A\end{array}$ \\
\hline 3 & $\begin{array}{c}32.78 \\
\text { A }\end{array}$ & $\begin{array}{c}32.56 \\
\text { A }\end{array}$ & $\begin{array}{c}31.56 \\
A\end{array}$ & $\begin{array}{c}31.41 \\
\text { A }\end{array}$ & $\begin{array}{c}30.83 \\
\text { A }\end{array}$ & $\begin{array}{c}31.36 \\
A\end{array}$ & $30.95 \mathrm{C}$ & $\begin{array}{c}30.90 \\
\text { B }\end{array}$ & $\begin{array}{c}30.38 \\
\text { A }\end{array}$ & $\begin{array}{c}29.75 \\
\text { A }\end{array}$ & $39.20 \mathrm{~A}$ \\
\hline 4 & $\begin{array}{c}33.30 \\
\mathrm{~A}\end{array}$ & $\begin{array}{c}32.91 \\
\mathrm{~A}\end{array}$ & $\begin{array}{c}31.85 \\
A\end{array}$ & $\begin{array}{c}31.63 \\
A\end{array}$ & $\begin{array}{c}30.55 \\
\mathrm{~A}\end{array}$ & $\begin{array}{c}32.68 \\
A\end{array}$ & $\begin{array}{c}31.20 \\
B C\end{array}$ & $\begin{array}{c}31.06 \\
\text { BA }\end{array}$ & $\begin{array}{c}30.93 \\
A\end{array}$ & $\begin{array}{c}30.41 \\
\mathrm{~A}\end{array}$ & $39.51 \mathrm{~A}$ \\
\hline 5 & $\begin{array}{c}33.13 \\
A\end{array}$ & $\begin{array}{c}32.53 \\
A\end{array}$ & $\begin{array}{c}31.75 \\
A\end{array}$ & $\begin{array}{c}31.61 \\
A\end{array}$ & $\begin{array}{c}29.81 \\
A\end{array}$ & $\begin{array}{c}32.66 \\
A\end{array}$ & $\begin{array}{c}32.26 \\
A B C\end{array}$ & $\begin{array}{c}31.45 \\
\text { BA }\end{array}$ & $\begin{array}{c}30.80 \\
A\end{array}$ & $\begin{array}{c}30.35 \\
A\end{array}$ & $39.16 \mathrm{~A}$ \\
\hline 6 & $\begin{array}{c}33.73 \\
\text { A }\end{array}$ & $\begin{array}{c}32.66 \\
\text { A }\end{array}$ & $\begin{array}{c}31.91 \\
\text { A }\end{array}$ & $\begin{array}{c}31.46 \\
\text { A }\end{array}$ & $\begin{array}{c}30.55 \\
\text { A }\end{array}$ & $\begin{array}{c}33.16 \\
\text { A }\end{array}$ & $\begin{array}{c}32.53 \\
\text { BA }\end{array}$ & $\begin{array}{c}31.30 \\
\text { BA }\end{array}$ & $\begin{array}{c}31.01 \\
\mathrm{~A}\end{array}$ & $\begin{array}{c}30.28 \\
\text { A }\end{array}$ & $39.23 \mathrm{~A}$ \\
\hline
\end{tabular}

Letras diferentes na mesma coluna $(P<0,05)$. T1E a T5E - temperaturas nos pontos do lado esquerdo do escroto; T1D a T5D - temperaturas nos pontos do lado direito do escroto; T1 - Cordão Espermático ; T2 - Terço Dorsal do Testículo; T3 - Terço Médio do Testículo; T4 - Terço Ventral do Testículo; T5 - Cauda do Epidídimo.

Para as variáveis WBTG, TA e TG, as maiores médias foram nas colheitas 4,5 e 6 . A maior UR obtida foi na colheita 3. Já para médias entre animais $(P<0,05)$ para $T A$, sendo que a maior temperatura foi $27,78^{\circ} \mathrm{C}$ (TABELA 3). 
Tabela 3. Valores médios, desvio padrão e análise de variância dos dados climáticos obtidos no local do experimento por termômetro de globo.

\begin{tabular}{c|cccc}
\hline & WBGT $^{1}$ & $\mathrm{TA}^{2}$ & $\mathrm{TG}^{3}$ & $\mathrm{UR}^{4}$ \\
\hline Média colheitas & & & & \\
1 & $20.42 \mathrm{C}$ & $24.73 \mathrm{~B}$ & $25.86 \mathrm{~B}$ & $57.32 \mathrm{CB}$ \\
2 & $20.48 \mathrm{C}$ & $23.98 \mathrm{~B}$ & $24.83 \mathrm{~B}$ & $56.13 \mathrm{CB}$ \\
3 & $22.85 \mathrm{~B}$ & $24.56 \mathrm{~B}$ & $24.68 \mathrm{~B}$ & $80.78 \mathrm{~A}$ \\
4 & $24.37 \mathrm{~A}$ & $29.91 \mathrm{~A}$ & $29.52 \mathrm{~A}$ & $58.23 \mathrm{CB}$ \\
5 & $24.81 \mathrm{~A}$ & $29.10 \mathrm{~A}$ & $29.48 \mathrm{~A}$ & $59.15 \mathrm{~B}$ \\
6 & $25.41 \mathrm{~A}$ & $30.80 \mathrm{~A}$ & $30.85 \mathrm{~A}$ & $52.56 \mathrm{C}$ \\
Média animais & & & & \\
1 & $22.40 \mathrm{~A}$ & $25.80 \mathrm{~B}$ & $26.16 \mathrm{~A}$ & $64.0 \mathrm{~A}$ \\
2 & $23.03 \mathrm{~A}$ & $27.23 \mathrm{BA}$ & $27.40 \mathrm{~A}$ & $59.58 \mathrm{~A}$ \\
3 & $23.30 \mathrm{~A}$ & $27.43 \mathrm{BA}$ & $27.61 \mathrm{~A}$ & $59.71 \mathrm{~A}$ \\
4 & $23.35 \mathrm{~A}$ & $27.61 \mathrm{BA}$ & $27.75 \mathrm{~A}$ & $60.35 \mathrm{~A}$ \\
5 & $23.46 \mathrm{~A}$ & $27.78 \mathrm{~A}$ & $27.75 \mathrm{~A}$ & $59.03 \mathrm{~A}$ \\
6 & $23.31 \mathrm{~A}$ & $26.41 \mathrm{BA}$ & $27.48 \mathrm{~A}$ & $60.30 \mathrm{~A}$ \\
\hline
\end{tabular}

Letras diferentes na mesma coluna $(\mathrm{P}<0,05) .{ }^{1}$ Temperatura global com termômetro úmido $\left({ }^{\circ} \mathrm{C}\right) ;{ }^{2} \mathrm{Temperatura}$ do ar $\left({ }^{\circ} \mathrm{C}\right) ;{ }^{3}$ Temperatura de globo preto $\left({ }^{\circ} \mathrm{C}\right) ;{ }^{4}$ Umidade relativa do ar $(\%)$.

Os níveis séricos de testosterona do presente trabalho foram de $253,51 \pm 112,24 \mathrm{ng} / \mathrm{dl}$, semelhantes ao resultado obtidos por Lezier (2004), indicando normalidade para este aspecto.

\section{DISCUSSÃO}

Os testículos dos bovinos devem ser mantidos abaixo da temperatura corporal central, para a produção de espermatozoides morfologicamente viáveis, normais e que a elevada temperatura ambiente está associada com a qualidade reduzida do sêmen (COULTER et al., 1988).

No presente trabalho, as variações observadas nas características qualitativas e quantitativas do sêmen entre as colheitas para motilidade, concentração, espermatozoides totais e espermatozoides viáveis totais $(P<0,05) \quad($ TABELA 1$)$, comparadas com as diferenças $(p<0,05)$ entre colheitas para as temperaturas do escroto, T1E, T2E, T3E, T4E, T1D, T2D, T3D e temperatura retal (TABELA 2) indicam que alterações nas temperaturas do escroto como na temperatura retal dos animais alteram a motilidade espermática, concentração, espermatozoides totais e espermatozoides viáveis totais. De acordo com Kastelic et al. (2001), uma moderada elevação da temperatura testicular em touros reduz de forma drástica a produção espermática, a motilidade progressiva e a quantidade de espermatozoides vivos por ejaculado, e aumenta a porcentagem de espermatozoides morfologicamente anormais. A elevação da temperatura testicular leva ao aumento do metabolismo e da demanda de oxigênio testicular, mas o fluxo sanguíneo testicular permanece estável 
e este aumento da demanda não é compensada, resultando em hipóxia e alterações na espermatogênese (SETCHELL, 2006).

As variações encontradas para as características do sêmen entre animais (TABELA 1) estão dentro dos padrões recomendados pelo CBRA (2013).

Elevadas temperaturas ambiente estão associadas com a redução da qualidade do sêmen (COULTER et al., 1988). As diferenças significativas $(P<0,05)$ encontradas neste estudo para WBGT, TA, TG e UR entre colheitas (TABELA 3) revela a variação climática entre as colheitas realizadas, influenciando na temperatura retal (TABELA 2) e características do sêmen (TABELA 1). Anchieta et al. (2005) e Chacur et al. (2013) observaram efeito negativo da estação chuvosa, a mais quente, sobre a motilidade espermática, tanto em touros zebus, quanto nos europeus mantidos em central de inseminação artificial.

No presente estudo, houve diferenças entre temperaturas do escroto entre colheitas (TABELA 2) para pontos específicos, sem alterar a morfologia espermática. Chacur et al. (2014) obtiveram $(P<0,05)$ para defeitos menores e defeitos totais comparando touros Nelore em diferentes estações do ano. Cook et al. (1994) observou que o aumento na temperatura escrotal está associado com o aumento dos defeitos espermáticos, e
Lunstra e Coulter (1997) descreveram que houve correlação positiva entre a temperatura do escroto e os defeitos de cauda dos espermatozoides.

\section{CONCLUSÃO}

As temperaturas da superfície do escroto mensuradas pela termografia escrotal por infravermelho influenciaram na qualidade do sêmen.

Os fatores climáticos: temperatura ambiente e umidade relativa do ar influenciaram na qualidade do sêmen.

Recomenda-se a adoção da termografia digital por infravermelho como exame complementar na realização do exame andrológico em touros Nelore, criados extensivamente.

\section{REFERÊNCIAS}

ANCHIETA, M.C.; VALE FILHO, V.R.; COLOSIMO, E. Descarte e congelabilidade do sêmen de touros de raças zebuínas e taurinas em central de inseminação artificial no Brasil. Arquivo Brasileiro de Medicina Vetrinária e Zootecnia, v.57, p.196-204, 2005. http://dx.doi.org/10.1590/S010209352005000200010

BRITO, L.F.; SILVA, A.E.; BARBOSA, R.T.; KASTELIC, J.P. Testicular thermoregulation in Bos indicus, crossbred and Bos taurus bulls: relationship with scrotal, testicular vascular cone and testicular morphology, and effects on semen quality and sperm production. Theriogenology, v.61, p.511-528, 2004. http://dx.doi.org/10.1016/S0093691X(03)00231-0 
CHACUR, M.G.M.; MIZUSAKI, K.T.; GABRIEL FILHO, L.R.A.; OBA, E.; RAMOS. A.A. Seasonal effects on semen and testosterone in zebu and taurine bulls. Acta Scientiae Veterinariae, v.41, p.1110, 2013.

CHACUR, M.G.M.; DOS REIS, J.D.A.; TAVARES, L.S.; SANCHES, K.; GUABERTO, L.; ALVES, V.C.; OBA, E.; RAMOS. A.A. Influência das épocas do ano na morfometria testicular e epididimária, características do sêmen e proteínas do sêmen em SDS-PAGE em zebus e taurinos. Acta Scientiae Veterinariae, v.42, p.1174, 2014.

CBRA (Colégio Brasileiro de Reprodução Animal). Manual para exame andrológico e avaliação de sêmen animal. 3. ed. Belo Horizonte: CBRA, 2013.

COOK, R.B.; COULTER, G.H.; KASTELIC J.P. The testicular vascular cone, scrotal thermoregulation and their relationship to sperm production and seminal quality in beef bulls. Theriogenology, v.41, p.653-671, 1994. http://dx.doi.org/10.1016/0093$\underline{691 \times(94) 90175-1}$

COULTER, G.H.; SERENGER, P.L.; BAILEY, D.R.C. Relationship of scrotal surface temperature measured by infrared thermography to subcutaneous and deep testicular temperature in the ram. Journal of Reproduction and Fertility, v.84, p.417-423, 1988.

\section{http://dx.doi.org/10.1530/jrf.0.0840417}

GABALDI, S.H.; WOLF, A. A importância da termorregulação testicular na qualidade do sêmen em touros. Ciência Agrária e Saúde, v.2, p.66-70, 2002.

HORN, M.M.; MORAES, J.C.F.; GALINA, C.S. Qualidade de sêmen de touros Aberdeen Angus e lbagé frente à degeneração testicular experimental. Archivos Latinoamericanos de Producción Animal, v.5, p.356-359, 1997.
KASTELIC, J.P. Understanding and evaluating bovine testes. Theriogenology, v.81, p.18-23, 2014.

http://dx.doi.org/10.1016/j.theriogenology.2 $\underline{013.09 .001}$

KASTELIC, J.P.; COOK, R.B.; PIERSON, R.A.; COULTER, G.H. Relationships among scrotal and testicular characteristics, sperm production, and seminal quality in 129 beef bulls. Canadian Journal of Veterinary Research, v. 65, p.111-115, 2001.

Lezier D.H. Avaliação da biometria testicular, concentração plasmática de hormônios e minerais em bovinos Nelore variedade mocha dos 12 aos 24 meses de idade. $76 f$. Botucatu, SP. Tese (Doutorado em Medicina Veterinária) - Programa de Pós-graduação em Reprodução Animal, Universidade Estadual Paulista. 2004.

LUNSTRA, D.D.; COULTER, G.H. Relationship between infrared temperature patterns and natural-mating fertility in beef bulls. Journal of Animal Science, v.75, p.767-774, 1997.

SAS. SAS/STAT user's guide. SAS Inst. Inc. Cary. NC. 2005.

SETCHELL, B.P. The effects of heat on the testes of mammals. Animal Reproduction, v.3, p.81-91, 2006. 